\title{
Fusarium keratitis as a growing problem - case report and literature review
}

\author{
Mateusz Winiarczyk ${ }^{1, A-F} \oplus$, Agnieszka Borkowska, ${ }^{1, B, D \oplus}$, Jerzy Mackiewicz ${ }^{1, E-F \oplus}$ \\ ${ }^{1}$ Department of Vitreoretinal Surgery, Medical University, Lublin, Poland \\ A - Research concept and design, B - Collection and/or assembly of data, C - Data analysis and interpretation, \\ $D$ - Writing the article, $E$ - Critical revision of the article, $F$ - Final approval of article \\ Winiarczyk M, Borkowska A, Mackiewicz J. Fusarium keratitis as a growing problem - case report and literature review. Ann Agric Environ Med. \\ 2021; 28(3): 409-413. doi: 10.26444/aaem/128682
}

\begin{abstract}
Introduction and objectives. With the rising number of contact lens users, there has been observed an increasing number of patients admitted to ophthalmology wards with severe keratitis, including those of fungal etiology. One of the most devastating specimens is Fusarium spp. If not diagnosed and treated from the onset of the symptoms, it can lead to severe visual loss, or even blindness.

Case report. This a retrospective case report of a 36-year-old pregnant female diagnosed with Fusarium keratitis, not responding to topical treatment. The problem is discussed and includes the most relevant literature review.

Results. Primary therapeutic keratoplasty is prone to failure due to an ongoing inflammation process in the eye, as happened in the described patient. Spread of the infection to the vitreous body necessitated the procedure of penetrating keratoplasty with lens removal, pars plana vitrectomy and silicone oil. After treatment, the inflammation resolved, and best corrected visual acuity at the 6 months follow-up was 0.5 (Snellen charts).

Conclusions. There is currently no treatment for Fusarium keratitis that would offer similar results to those observed in bacterial infection treated early with antibiotics. As systemic therapy with antifungal agents is not an option in pregnant women, they are even more of a challenge. Diagnosis is quite often delayed, leading to deeper infiltration of the cornea, sometimes with vitreous involvement. For such cases, a combined approach is proposed with vitrectomy, lens removal and penetrating keratoplasty. Early surgical intervention may rescue some of the eyes non-responsive to topical and systemic treatment.
\end{abstract}

Key words

fungal keratitis, Fusarium keratitis, keratoplasty, vitrectomy, infection

\section{INTRODUCTION}

Fungal keratitis is a progressive ocular infection, that can cause corneal perforation and involvement of the vitreous body, leading to severe visual impairment, blindness, or even removal of the eyeball. With the rising number of contact lens wearers, it is becoming an increasing burden in developed countries, and is still responsible for almost $40 \%$ of all ulcers in tropical climate [1]. Fusarium spp. belongs to a mould group and can cause a potentially fulminant course of disease. Risk factors of Fusarium keratitis are contact lenses use, trauma - especially contaminated with organic material, immunosuppression, and therapy with corticosteroids [2, $3,4]$. One of the best documented outbreaks of Fusarium keratitis associated with contact lens use, occurred in the United States in 2005-2006, during which 250 cases were reported due to contamination of multipurpose contact lens disinfecting solutions [5].

One of the most important issues in Fusarium keratitis is its early recognition. When suspected, immediate corneal scraping for culture should be performed, with administration of topical and systemic antifungal agents. Even then, Fusarium spp. infection can progress, as they demonstrate high resistance to most of the antifungal drugs [6], and a surgical intervention has to be considered. In some cases, the corneal infiltrate penetrates deeper through the

Address for correspondence: Jerzy Mackiewicz, Department of Vitreoretinal Surgery, Medical University of Lublin, Chmielna 1, 20-079, Lublin, Poland E-mail: jerzymackiewicz@umlub.p

Received: 25.05.2020; accepted: 21.10.2020; first published: 23.11.2020 cornea into the vitreous, causing panophthalmitis. In those severe cases, enucleation may be eventually needed $[7,8]$.

\section{CASE REPORT}

The case is presented of a 36-year-old pregnant $\left(25^{\text {th }}\right.$ week $)$ female with no comorbidities, but with a complicated course of the disease, and treatment outcome. The patient was admitted to the Ophthalmic Clinic with a history of pain, photophobia and ocular hyperaemia lasting for two weeks. There was no history of trauma. She was a contact lens wearer but did not use them for two weeks before the onset of the symptoms, as she was immobilized in bed due to knee surgery. After developing the first ocular manifestati, she was treated in the local ophthalmology department with various combinations of moxifloxacin, dexamethasone, tropicamide and ganciclovir. On referral, she presented with confluent, disciform, almost full-thickness corneal infiltrate, confirmed in anterior OCT examination (SS-1000 Casia OCT, Tomey) (Fig. 1). Her best corrected visual acuity (BCVA) was hand motion, with intraocular pressure (IOP) of $12 \mathrm{mmHg}$. Immediate corneal scraping samples for microbiological culture were taken, and topical treatment was introduce: voriconazole (Vfend, Pfizer, Belgium) 5x, levofloxacin 4x (Oftaquix, Santen, Finland), PHMB 5x (Brolene, Sanofi, UK), Tropicamide 3x. (Tropicamidum WZF 1\%, Polfa SA, Poland). Because of pregnancy, it was not possible to administer systemic treatment. Unfortunately, confocal microsope examination, could not be performed due to the lack of such an instrument. 
When no improvement was seen after four days, and with a growing risk of corneal perforation, the decision was made to proceed with penetrating therapeutic keratoplasty. The surgery waperformede under peribulbar anaesthesia due to the pregnancy. After the Flierienga ring was sewn on, a host graft of $8.00 \mathrm{~mm}$ diameter was excised and replaced by a $8.25 \mathrm{~mm}$ donor cornea. Iridectomy was performed, and graft s stabilized with 10/0 interrupted sutures. Host tissue was cut in half and sent both for microbiological and histopathological examination.

Three days after surgery the graft was almost fully epithelized, and there were no signs of recurrence (Fig. 2 ). The patient was discharged home with significant improvement in visual acuity - BCVA 0.15 and IOP of $6 \mathrm{mmHg}$, with topical treatme: voriconazole (Vfend, Pfizer, Belgium) 5x, ofloxacin (Vigamox, Novartis, Poland) 5x, tropicamide (Tropicamidum WZF 1\%, Polfa SA, Poland) 3x, dexamethasone (Dexamethason WZF 1\%, Polfa SA, Poland) 6x, and lubrication (Hexoftyal, Melleson Pharma, Holland).

After 11 days, the patient was readmitted to ther clinic with significant eye pain and visual acuity drop. Her BCVA was 0.05 , and $\mathrm{IOP}=14 \mathrm{mmHg}$. Hyperaemia, corneal graft oedema, infiltrates around sutures, and inflammation in the anterior chamber was seen in the clinical examination, with no signs of vitreous involvement in ultrasound B examination. At the same time, the final microbiological report showed Fusarium spp. growth, with a negative Acanthamoeba result. Intensive antifungal topical treatment with $5 \%$ natamycin (Natacyn, Alcon, USA) and voriconazole (Vfend, Pfizer, Belgium, 1\% solution) every hour was introduced with levofloxacin (Oftaquix, Santen, Finland) 5x, tropicamide (Tropicamidum WZF 1\%, Polfa SA, Poland) 3x, and artificial tears (Hylo-Comod, Ursapharm, Germany) (Thealoz Duo, Thea, France). Steroids were discontinued immediately after admission. During the next four days, worsening of the corneal condition with hypopyon formation was observed, and BCVA dropped in counting fingers from $1 \mathrm{~m}$. The anterior chamber of the eye was additionally washed three times twice with voriconazole and once with gentamycin in the course of seven days.

Two weeks after admission, hyperreflective vitreous in ultrasound examination was observed, suggesting panophthalmitis. Clinical presentation during readmission, with ultrasound examination of inflamed vitreous body and hypopyon were observed in the anterior chamber of the eye (Fig. 3). Due to the lack of significant improvement, the decision was made to proceed with the surgical procedure of keratoplasty combined with phacovitrectomy. Under general anaesthesia, the oedematous corneal graft was replaced with temporary keratoprosthesis, followed by phacoemulsification of the lens and intraocular lens (IOL) implantation. 23G pars plana vitrectomy was performed with removal of the inflamed vitreous body. During the procedure, a vitreous body sample was obtained for microbiological culture. Afterwards, the keratoprosthesis was removed, and new graft was stabilized with 10/0 interrupted Nylon sutures. Silicone oil was installed in the end of the surgery

Postoperative treatment consisted of 5\% natamycin (Natacyn, Alcon, USA) with voriconazole (Vfend, Pfizer, Belgium, 1\% solution) each hour, levofloxacin (Oftaquix, Santen, Finland) 4x, tropicamide (Tropicamidum WZF $1 \%$, Polfa SA, Poland) 3x, artificial tears (Hylo-Comod, Ursapharm, Germany) (Thealoz Duo, Thea, France) 5x.

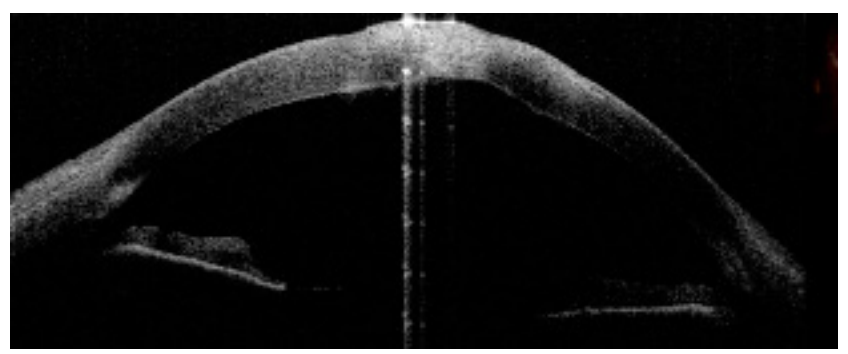

Figure 1. Preoperative state, showing almost full-thickness infiltrate of the central cornea visualized in anterior OCT examination

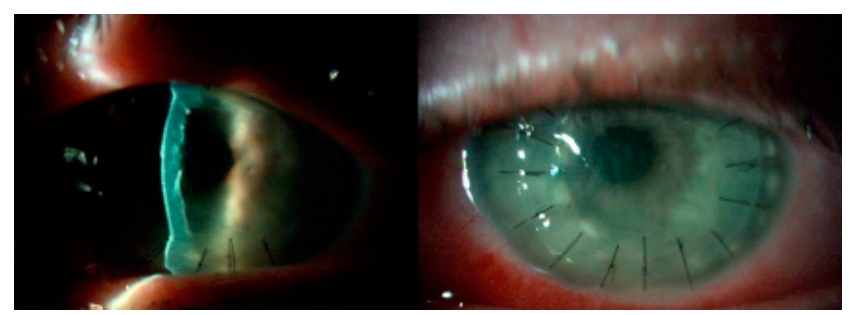

Figure 2. Clinical presentation after first corneal transplant. Clear cornea with no signs of recurrence is visible

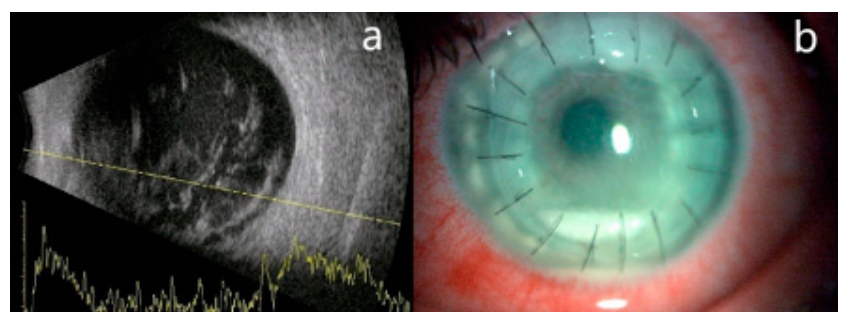

Figure 3. Clinical condition before second surgery. a) ultrasound examination showing hyperreflective echo suggesting of endophthalmitis; b) slit lamp examination with visible hypopyon

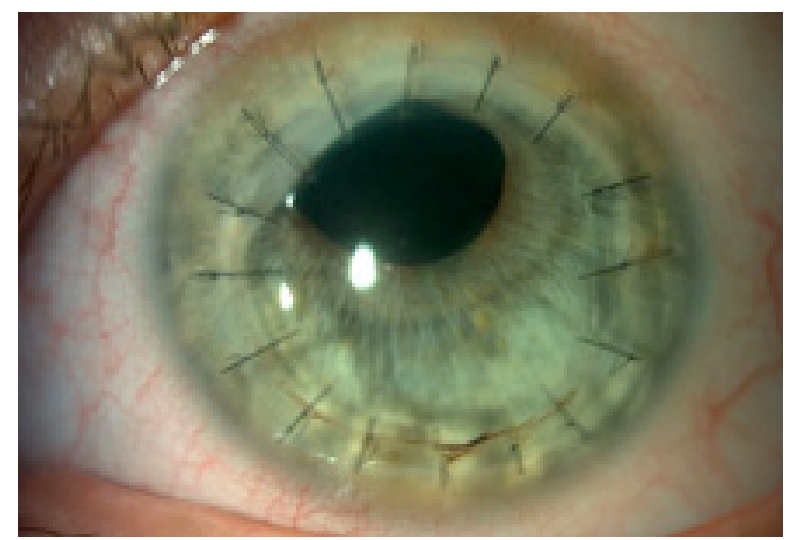

Figure 4. Clinical state during follow-up visit after 6 months from onset of the disease, clear corneal graft

In the following five days, BCVA remained at fingers count from $50 \mathrm{~cm}$, and the IOP was $17 \mathrm{mmHg}$. The patient was discharged with continuation of topical treatment. Steroids (dexamethasone $3 \mathrm{x}$ ) were added one month later, after no signs of recurrence were observed.

At six-months follow-up, the corneal graft was clear, there are no signs of inflammation (Fig. 4), the BCVA is 0.5 Snellen - pinhole (PH), with IOP of $16 \mathrm{mmH}$. The patient was satisfied with the result of treatment. 


\section{DISCUSSION}

Risk factors. In the developing countries, Fusarium keratitis remains one of the main causes of infectious keratitis $[9,10]$ and is caused mainly by organic contamination of the corneal traumatic wound, usually during gardening in the home. Therefore, due to everyday exposure to organic material, the inhabitants of rural areas are in the group at higher risk of infection than those from the urban environment. Lower financial and educational status also play important roles, as some patients may choose self-medication or delay an ophthalmologist appointment. In a large epidemiological study, significant disparity in the risk of developing infectious keratitis was shown between rural and urban inhabitants [11]. It is suggested that greater effort should be focused on the education of patients from agricultural areas. Another and growing risk factor is an increasing number of contact lens users $[2,3,12]$. Interestingly, in the described case, the patient had suspended her CT use two weeks beforethen onset of symptoms due to immobilization in bed due to a pulled leg ligament. For the same reason, she also denied any trauma. The mechanism by which Fusarium contaminated material came into contact with her eye remains unclear, but the long history of keratitis development should always be concerned as a potential fungal etiology $[1,13]$.

Diagnostics. The first problem faced by a physician in the course of fungal keratitis is the diagnostic process. This may take up to two weeks for the growth in microbiology culture, delaying treatment. Clinical features of the ulcer should cause concern about its fungal etiology - satellite lesions, feathery borders, intact epithelium, and usually slower progression than observed in bacterial, or even Acanthamoeba infection [14]. If the antifungal treatment is not promptly started, Fusarium hyphae would penetrate deeper through the stroma, producing mycotoxins and proteolytic enzymes that cause corneal melting. For those reasonsa superficial smear from the conjunctival sack or cornea is insufficient for diagnosis; corneal scraping, or even biopsy with a needle, spatula, or a dermatological punch is recommended [14, 15]. PCR testing can reduce the time needed for fungal identification, but standard culture on Sabouraud agar is still a golden standard in diagnosis $[15,16]$. The usual time for the first growth in the culture was reported to be $72 \mathrm{~h}$ in $83 \%$ of cultures, and seven days in $97 \%$ [14]. In vivo confocal microscopy is a viable option for fast and reliable diagnosis of fungal keratitis, as shown in previous studies $[17,18,19]$.

In the authors' experience, a significant number of cases with no growth in microbiological laboratory is observed, which was the case in the presented patient. It was only after culture obtained from the first keratoplasty material that fungal growth was observed and its drug sensitivity determined - 15 days after the sample was taken.

Medical therapy. The medical treatment of Fusarium keratitis is challenging as it develops an intrinsic resistance to the majority of antifungal agents [6]. Azoles (voriconazole, fluconazole, ketoconazole, posaconazole, itraconazole) and polyenes (natamycin, amphotericin B). Topical 1\% Voriconazole and $5 \%$ natamycin are currently the most potent drugs in Fusarium treatment. Both agents have limited ability to penetrate the corneal epithelium; therefore, some authors suggest repeatable epithelial debridement before eyedrop application [13]. Synergistic effect has been pursued in several studies, combining both topical natamycin with voriconazole $[14,20]$, topical natamycin with systemic ketoconazole [21], topical and systemic voriconazole [22], voriconazole with micafungin [23]. Although single case reports show some promising results, they were never replicated in larger clinical trials. Most recent studies suggest that $5 \%$ natamycin is a drug of choice, although it is not commercially available in Poland $[20,21,24,25,26]$. Due to pregnancy, in the presented patient treatment was limited to topical medications; therefore, medication commenced with voriconazole, with natamycin added as soon as was possible to import it from a foreign pharmacy. An additional value of voriconazole is that it can be also injected intrastromally and used for anterior chamber washout, although most studies show no benefit of adding it to sole natamycin, or superiority over topical application of sole voriconazole $[26,27,28,29]$.

With good results obtained in bacterial keratitis [30, 31] and promising preliminary studies, corneal cross-linking (CXL) has been proposed as an adjuvant therapy in fungal keratitis [32, 33, 34]. Unfortunately, those results were not replicated in clinical trials, showing no added value of CXL in the traditional therapy of fungal keratitis, and with an even inferior effect $[35,36]$. As this data is confusing, it may be hypothesized, that CXL may have role limited to early fungal keratitis, as in moderate and advanced stages the infiltrate spreads deeper into the corneal stroma, making CXL ineffective.

Surgical approach. When topical and systemic treatment fails, and no improvement or worsening of the infiltrate is observed, therapeutic keratoplasty should be performed. There is no consensus about an exact timepoint or clinical features that would qualify for corneal transplant, but general agreement seems to shift to 'as soon as necessary'. If surgical treatment is delayed for too long, fungus may penetrate through the Descemet's membrane (DM) and spread into the vitreous, causing panophthalmitis $[7,13,16]$.

Deep anterior lamellar keratoplasty (DALK) is a tempting idea if a therapeutic keratoplasty has to be performed in Fusarium keratitis patients. As they are often young and otherwise healthy, their corneal endothelium is of far better quality than one can expect after penetrating keratoplasty (PK). Therefore, preserving the endothelium with the Descemet membrane, with total resection of the infiltrate was tried in several studies [37, 38, 39, 40, 41]. In all of them, DALK was considered as a viable alternative for PK, with considerable advantages of lower immune rejection and graft dehiscence, but limited by the depth of the fungal infiltrate - i.e. Sabatino et al. did not consider it in ulcers deeper than $300 \mu \mathrm{m}$ [39]. The biggest disadvantage of this approach is the difficult surgical technique, and risk of leaving some fungal hyphae unnoticed in the deeper layers of the cornea, but this can be minimized with careful patient selection.

The most common surgical procedure for non-responsive fungal keratitis is penetrating keratoplasty, where fullthickness corneal graft is removed and replaced by healthy donor tissue. This approach offers the highest chance of total removal of the lesion, although it is also associated with serious complications [42]. If the corneal perforation, diffuse and deep melting, or vitreous involvement is present, $\mathrm{PK}$ is the treatment of choice associated with satisfactory results. Unfortunately, a high percentage of complications after PK 
in fungal keratitis is observed - immune graft rejection, recurrence, or secondary glaucoma $[42,43,44,45]$. In some cases, the disease progresses to the endophthalmitis stage which usually requires immediate vitrectomy, but even then, those are eyes with high risk of enucleation [7, 46, 47, 48].

In the presented case, after primary penetrating keratoplasty failed, the patient required combined surgical treatment of temporary keratoprosthesis, lens phacoemulsification with IOL implantation, vitrectomy with silicone oil tamponade, and penetrating keratoplasty. All e signs of infection were removed and the inflammation controlled. In the follow up visit after 6 months, she restored BCVA of $0.5 \mathrm{PH}$, and no complications.

\section{CONCLUSION}

Fusarium induced keratitis is a potentially sight-threatening condition which should be quickly diagnosed and aggressively treated. Pregnant female patients are at higher risk of a severe course of the disease, as they cannot be treated with systemic antifungal agents. The described patient recovered only after a second, complex surgery and showed no signs of recurrence in the next six months of the follow-up. The presented study demonstrates how complicated the treatment can be in such cases

The authors would like to highlight that early surgical intervention in aggressive fungal keratitis in such patients should be considered much earlier than in regular cases.

\section{REFERENCES}

1. Garg P, Roy A, Roy S. Update on fungal keratitis. Curr Opin Ophthalmol. 2016; 27(4): 333-339.

2. Gower EW, Keay LJ, Oechsler RA, Iovieno A, Alfonso EC, Jones DB, et al. Trends in fungal keratitis in the United States, 2001 to 2007. Ophthalmology. 2010; 117(12): 2263-7.

3. Bullock JD, Elder BL, Khamis HJ, Warwar RE. Effects of Time, Temperature, and Storage Container on the Growth of Fusarium Species: Implications for the Worldwide Fusarium Keratitis Epidemic of 2004-2006. Arch Ophthalmol. 2011; 129(2): 133-6.

4. Gopinathan U, Garg P, Fernandes M, Sharma S, Athmanathan S, Rao GN. The Epidemiological Features and Laboratory Results of Fungal Keratitis: A 10-Year Review at a Referral Eye Care Center in South India. Cornea. 2002; 21(6): 555-559.

5. Epstein AB. In the aftermath of the Fusarium keratitis outbreak: What have we learned? Clin Ophthalmol Auckl NZ. 2007; 1(4): 355-66.

6. Al-Hatmi AMS, Meis JF, de Hoog GS. Fusarium: Molecular Diversity and Intrinsic Drug Resistance. PLoS Pathog. 2016; 12(4). Dostępne na: https://www.ncbi.nlm.nih.gov/pmc/articles/PMC4824402/

7. Dursun D, Fernandez V, Miller D, Alfonso EC. Advanced Fusarium Keratitis Progressing to Endophthalmitis. Cornea. 2003; 22(4): 300-303.

8. Fusarium Keratitis in Germany. J Clin Microbiol. 2017 Oct; 55(10): 2983-2995. doi: 10.1128/JCM.00649-17 https://jcm.asm.org/ content/55/10/2983.short

9. Bharathi MJ, Ramakrishnan R, Meenakshi R, Padmavathy S, Shivakumar C, Srinivasan M. Microbial Keratitis in South India: Influence of Risk Factors, Climate, and Geographical Variation. Ophthalmic Epidemiol. 2007; 14(2): 61-9.

10. Leck AK, Thomas PA, Hagan M, Kaliamurthy J, Ackuaku E, John $\mathrm{M}$, et al. Aetiology of suppurative corneal ulcers in Ghana and south India, and epidemiology of fungal keratitis. Br J Ophthalmol. 2002; 86(11): 1211-5.

11. Cao J, Yang Y, Yang W, Wu R, Xiao X, Yuan J, et al. Prevalence of infectious keratitis in Central China. BMC Ophthalmol. 2014; 14(1): 43.

12. Alipour F, Khaheshi S, Soleimanzadeh M, Heidarzadeh S, Heydarzadeh S. Contact Lens-related Complications: A Review. J Ophthalmic Vis Res. 2017; 12(2): 193-204.

13. Tuli SS. Fungal keratitis. Clin Ophthalmol Auckl NZ. 2011; 5: 275-9.
14. Ansari Z, Miller D, Galor A. Current Thoughts in Fungal Keratitis: Diagnosis and Treatment. Curr Fungal Infect Rep. 2013; 7(3): 209-18.

15. Maharana PK, Sharma N, Nagpal R, Jhanji V, Das S, Vajpayee RB. Recent advances in diagnosis and management of Mycotic Keratitis. Indian J Ophthalmol. 2016; 64(5): 346-57.

16. Wu J, Zhang W-S, Zhao J, Zhou H-Y. Review of clinical and basic approaches of fungal keratitis. Int J Ophthalmol. 2016; 9(11): 1676-83.

17. Chidambaram JD, Prajna NV, Palepu S, Lanjewar S, Shah M, Elakkiya $S$, et al. Cellular morphological changes detected by laser scanning in vivo confocal microscopy associated with clinical outcome in fungal keratitis. Sci Rep. 06 2019; 9(1): 8334.

18. Chidambaram JD, Prajna NV, Larke N, Macleod D, Srikanthi P, Lanjewar S, et al. In vivo confocal microscopy appearance of Fusarium and Aspergillus species in fungal keratitis. Br J Ophthalmol. 2017; 101(8): 1119-23.

19. Tabatabaei SA, Soleimani M, Tabatabaei SM, Beheshtnejad AH, Valipour N, Mahmoudi S. The use of in vivo confocal microscopy to track treatment success in fungal keratitis and to differentiate between Fusarium and Aspergillus keratitis. Int Ophthalmol. 2020; 40(2): 483-91.

20. Prajna NV, Mascarenhas J, Krishnan T, Reddy PR, Prajna L, Srinivasan $M$, et al. Comparison of Natamycin and Voriconazole for the Treatment of Fungal Keratitis. Arch Ophthalmol. 2010; 128(6): 672-8.

21. Rajaraman R, Bhat P, Vaidee V, Maskibail S, Raghavan A, Sivasubramaniam S, et al. Topical 5\% Natamycin With Oral Ketoconazole in Filamentous Fungal Keratitis: A Randomized Controlled Trial. Asia-Pac J Ophthalmol. 2015; 4(3): 146-150.

22. Klont RR, Eggink CA, Rijs AJMM, Wesseling P, Verweij PE. Successful Treatment ofFusarium Keratitis with Cornea Transplantation and Topical and Systemic Voriconazole. Clin Infect Dis. 2005; 40(12): e110-2.

23. Heyn K, Tredup A, Salvenmoser S, Müller F-MC. Effect of Voriconazole Combined with Micafungin against Candida, Aspergillus, and Scedosporium spp. and Fusarium solani. Antimicrob Agents Chemother. 2005; 49(12): 5157-9.

24. Prajna NV, Krishnan T, Mascarenhas J, Rajaraman R, Prajna L, Srinivasan M, et al. The Mycotic Ulcer Treatment Trial: A Randomized Trial Comparing Natamycin vs Voriconazole. JAMA Ophthalmol. 2013; 131(4): 422-9.

25. Pradhan L, Sharma S, Nalamada S, Sahu SK, Das S, Garg P. Natamycin in the treatment of keratomycosis: Correlation of treatment outcome and in vitro susceptibility of fungal isolates. Indian J Ophthalmol. 2011; 59(6): 512-4.

26. Sharma N, Chacko J, Velpandian T, Titiyal JS, Sinha R, Satpathy G, et al. Comparative evaluation of topical versus intrastromal voriconazole as an adjunct to natamycin in recalcitrant fungal keratitis. Ophthalmology. 2013; 120(4): 677-81.

27. Kalaiselvi G, Narayana S, Krishnan T, Sengupta S. Intrastromal voriconazole for deep recalcitrant fungal keratitis: a case series. Br J Ophthalmol. 2015; 99(2): 195-8.

28. Niki M, Eguchi H, Hayashi Y, Miyamoto T, Hotta F, Mitamura Y. Ineffectiveness of intrastromal voriconazole for filamentous fungal keratitis. Clin Ophthalmol Auckl NZ. 2014; 8: 1075-9.

29. Mycotic Antimicrobial Localized Injection: A Randomized Clinical Trial Evaluating Intrastromal Injection of Voriconazole. Ophthalmology. 2019; 126(8): 1084-9.

30. Papaioannou L, Miligkos M, Papathanassiou M. Corneal Collagen Cross-Linking for Infectious Keratitis: A Systematic Review and MetaAnalysis. Cornea. 2016; 35(1): 62-71.

31. Makdoumi K, Mortensen J, Crafoord S. Infectious Keratitis Treated With Corneal Crosslinking. Cornea. 2010; 29(12): 1353-1358.

32. Li Z, Jhanji V, Tao X, Yu H, Chen W, Mu G. Riboflavin/ultravoilet light-mediated crosslinking for fungal keratitis. Br J Ophthalmol. 2013; 97(5): 669-71.

33. Galperin G, Berra M, Tau J, Boscaro G, Zarate J, Berra A. Treatment of Fungal Keratitis From Fusarium Infection by Corneal Cross-Linking. Cornea. 2012; 31(2): 176-180.

34. Wei A, Wang K, Wang Y, Gong L, Xu J, Shao T. Evaluation of corneal cross-linking as adjuvant therapy for the management of fungal keratitis. Graefes Arch Clin Exp Ophthalmol Albrecht Von Graefes Arch Klin Exp Ophthalmol. 2019; 257(7): 1443-52.

35. Uddaraju M, Mascarenhas J, Das MR, Radhakrishnan N, Keenan JD, Prajna L, et al. Corneal Cross-linking as an Adjuvant Therapy in the Management of Recalcitrant Deep Stromal Fungal Keratitis: A Randomized Trial. Am J Ophthalmol. 2015; 160(1): 131-134. e5.

36. Cross-Linking-Assisted Infection Reduction: A Randomized Clinical Trial Evaluating the Effect of Adjuvant Cross-Linking on Outcomes in Fungal Keratitis. Ophthalmology. 2020; 127(2): 159-66. 
37. Arora T, Arora S, Sharma V. Early Deep Anterior Lamellar Keratoplasty (DALK) for Acanthamoeba Keratitis-How to Define Early? Cornea. 2016; 35(6): e14.

38. Gao H, Song P, Echegaray JJ, Jia Y, Li S, Du M, et al. Big Bubble Deep Anterior Lamellar Keratoplasty for Management of Deep Fungal Keratitis. J Ophthalmol. Hindawi; 2014. s. e209759. https://www. hindawi.com/journals/joph/2014/209759/

39. Sabatino F, Sarnicola E, Sarnicola C, Tosi GM, Perri P, Sarnicola V. Early deep anterior lamellar keratoplasty for fungal keratitis poorly responsive to medical treatment. Eye. 2017; 31(12): 1639-46.

40. Sarnicola E, Sarnicola C, Sabatino F, Tosi GM, Perri P, Sarnicola V. Early Deep Anterior Lamellar Keratoplasty (DALK) for Acanthamoeba Keratitis Poorly Responsive to Medical Treatment: Cornea. 2016; 35(1): $1-5$.

41. Uchio E, Saeki Y, Tsukahara-Kawamura T, Kadonosono K, Ozaki H. Clinical outcome after air-assisted manual deep anterior lamellar keratoplasty for fungal keratitis poorly responsive to medical treatment. Clin Ophthalmol Auckl NZ. 2019; 13: 1913-9.
42. Ti S-E, Scott JA, Janardhanan P, Tan DTH. Therapeutic Keratoplasty for Advanced Suppurative Keratitis. Am J Ophthalmol. 2007; 143(5): 755-762. e2.

43. Chen W-L, Wu C-Y, Hu F-R, Wang I-J. Therapeutic penetrating keratoplasty for microbial keratitis in Taiwan from 1987 to 2001. Am J Ophthalmol. 2004; 137(4): 736-43.

44. Lalitha P, Prajna NV, Kabra A, Mahadevan K, Srinivasan M. Risk Factors for Treatment Outcome in Fungal Keratitis. Ophthalmology. 2006; 113(4): 526-30.

45. Xie L, Zhai H, Shi W. Penetrating Keratoplasty for Corneal Perforations in Fungal Keratitis. Cornea. 2007; 26(2): 158-162.

46. Edelstein SL, Akduman L, Durham BH, Fothergill AW, Hsu HY. Resistant Fusarium Keratitis Progressing to Endophthalmitis. Eye Contact Lens. 2012; 38(5): 331-335.

47. Rosenberg KD, Flynn HW, Alfonso EC, Miller D. Fusarium Endophthalmitis Following Keratitis Associated With Contact Lenses. Ophthalmic Surg Lasers Imaging Retina. 2006; 37(4): 310-3.

48. Proença-Pina J, Ssi Yan Kai I, Bourcier T, Fabre M, Offret H, Labetoulle M. Fusarium keratitis and endophthalmitis associated with lens contact wear. Int Ophthalmol. 2010; 30(1): 103-7. 